\title{
Temperaturmessung gemäß der Internationalen Temperaturskala von 1990
}

\author{
Steffen, Rudtsch und Joachim, Fischer \\ Physikalisch-Technische Bundesanstalt (PTB), Abbestr. 2-12, 10587 Berlin \\ Tel.: 0303481 7650, e-mail: steffen.rudtsch@ptb.de
}

\section{Einleitung}

Die Temperatur gehört zu den am häufigsten gemessenen physikalischen Größen. Sie ist eine entscheidende Kenngröße beim Klimawandel, in der medizinischen Diagnostik und Therapie, beim Energieumsatz in Motoren und in Kraftwerken. Den Ablauf von Lebensprozessen bestimmt sie ebenso wie sie als Steuergröße in der Chemie wirkt. Aufgrund unterschiedlichster messtechnischer Anforderungen wird für Temperaturmessungen eine Vielzahl an physikalischen Prinzipien angewendet. Bei industriellen Anwendungen geht es oft darum, ein langjährig genutztes und bewährtes Messverfahren dahingehend zu qualifizieren, dass bestimmte Normen eingehalten bzw. eine Rückführung der Ergebnisse mit vorgegebenen Messunsicherheiten sichergestellt werden muss. Hierbei ist es sehr vorteilhaft, wenn bereits in der Phase der Entwicklung und dem Aufbau von Temperaturmesssytemen für industrielle Anwendungen eine Analyse der wesentlichen Beiträge zur Messunsicherheit erfolgt und die Vorgehensweise bei der metrologischen Rückführung erarbeitet wird.

\section{Thermodynamische Temperatur und die Notwendigkeit von Temperaturskalen}

Die Temperatureinheit ist durch zwei Fixpunkte, den absoluten Nullpunkt und den Tripelpunkt des Wassers (Abb.1), sowie die Festlegung einer Teilung dieses Bereiches definiert. Hieraus ergibt sich die derzeit gültige Definition: „Das Kelvin, die Einheit der thermodynamischen Temperatur, ist der 273,16te Teil der thermodynamischen Temperatur des Tripelpunktes des Wassers." [1]

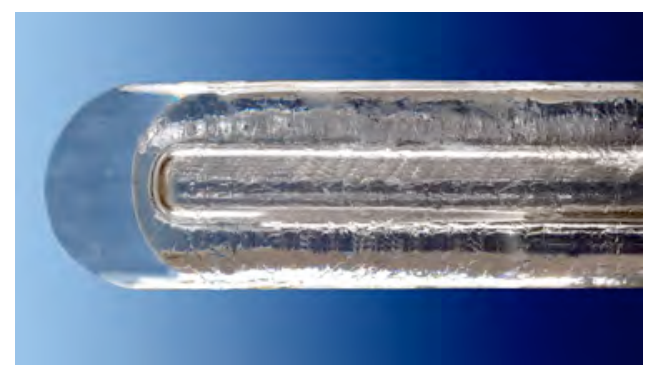

Abb. 1 Phasengrenzfläche flüssig-fest bei einer Wassertripelpunktszelle

Da die Temperatur eine intensive Größe ist, müssen für deren Darstellung sogenannte Primärthermometer zur direkten Bestimmung thermodynamischer Temperaturen verwendet weden. Hierbei handelt es sich z.B. um spezielle Gasthermometer, Strahlungsthermometer, Johnson-Rauschthermometer oder Thermometer die auf der thermischen Verbreiterung von Doppler-Linien beruhen. In der Regel sind Messungen mit derartigen Thermometern jedoch sehr aufwendig und langwierig, so dass sie für Aufgaben im Bereich der technischen Temperaturmessung nicht infrage kommen. Dafür verwendet man sekundäre Thermometer, bei denen der Vorteil einer leichteren 
Handhabbarkeit dadurch erkauft wird, dass man diese an verschiedenen Stützstellen (Temperaturfixpunkten) kalibrieren muss. Hierzu nutzt man aus, dass thermodynamische Gleichgewichtszustände zwischen den Phasen reiner Substanzen vergleichsweise einfach und mit sehr hoher Reproduzierbarkeit realisiert werden können.

\section{Die Internationale Temperaturskala von 1990 (ITS-90) und deren Fixpunkte}

Die derzeit gültige Internationale Temperaturskala von 1990 (ITS-90) wurde 1989 vom Internationalen Komitee für Maß und Gewicht (CIPM) verabschiedet. Deren untere Grenze liegt bei $0,65 \mathrm{~K}$. Eine obere Temperaturgrenze ist nicht direkt festgelegt, sondern ergibt sich aus der Anwendbarkeit des Planck'schen Strahlungsgesetzes. Die Kalibrierung der verwendeten Thermometer erfolgt an den definierenden Fixpunkten (Abb. 1), denen bestimmte Temperaturwerte zugeschrieben sind (Tab. 1).
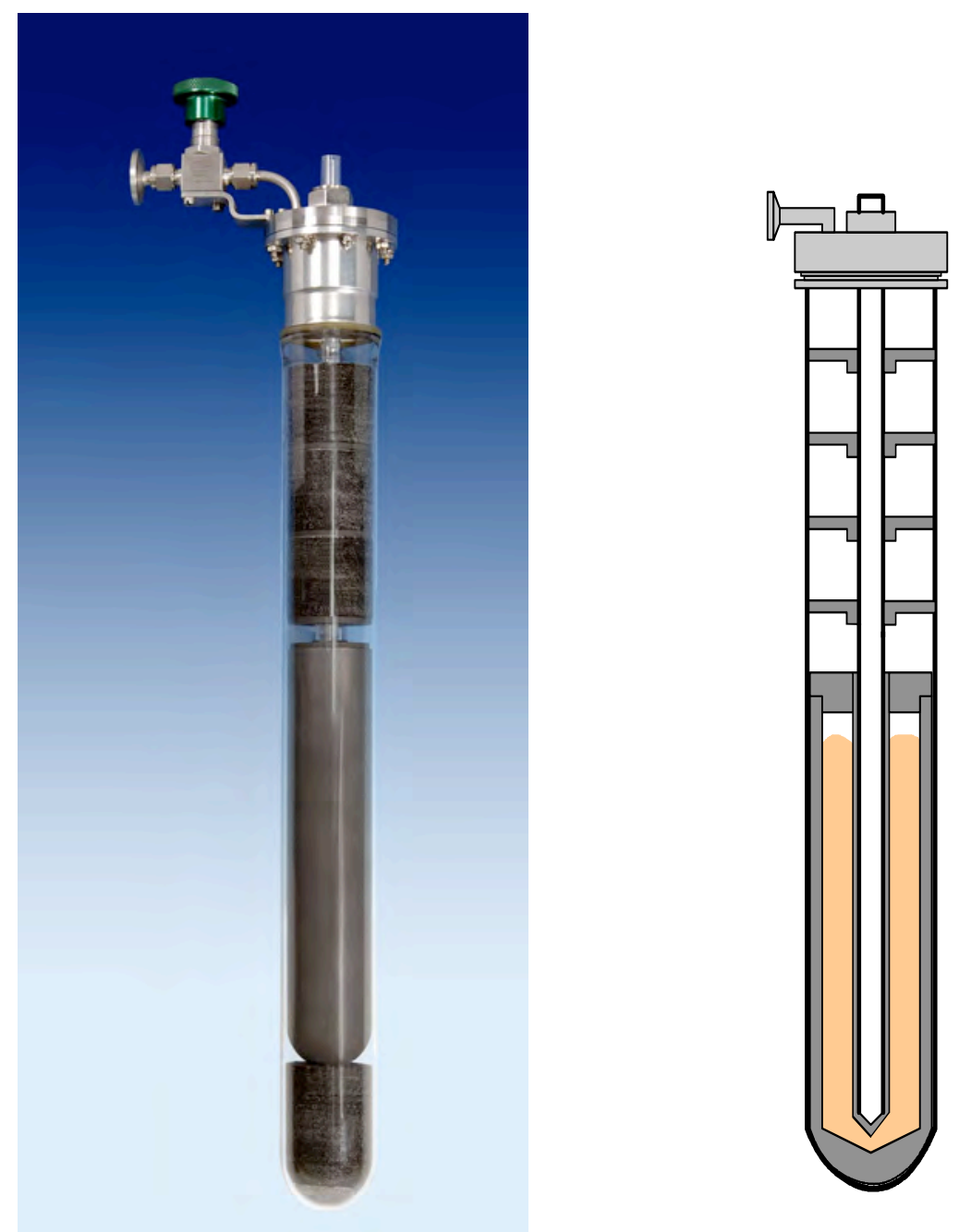

Abb. 1 Typischer Aufbau einer Fixpunktzelle für den Temperaturbereich von $156{ }^{\circ} \mathrm{C}$ bis $962{ }^{\circ} \mathrm{C}$ 
Tabelle1 Definierende Fixpunkte der ITS-90

\begin{tabular}{|c|c|c|c|c|}
\hline Substanz & Zustand & $\boldsymbol{T}_{\mathbf{9 0}} / \mathbf{K}$ & $\boldsymbol{u}_{\text {thermodyn }} / \mathbf{m K}$ & $\boldsymbol{u}_{\text {NMls }} / \mathbf{m K}$ \\
\hline $\mathrm{He}$ & $\mathrm{Dd}$ & $3-5$ & 0,3 & 0,2 \\
\hline $\mathrm{e}-\mathrm{H}_{2}$ & $\mathrm{Tp}$ & 13,8033 & 0,4 & 0,2 \\
\hline $\mathrm{e}-\mathrm{H}_{2}$ & $\mathrm{Dd}$ & $\approx 17$ & 0,5 & 0,2 \\
\hline $\mathrm{e}-\mathrm{H}_{2}$ & $\mathrm{Dd}$ & $\approx 20,3$ & 0,5 & 0,3 \\
\hline $\mathrm{Ne}$ & $\mathrm{Tp}$ & 24,5561 & 0,5 & 0,2 \\
\hline $\mathrm{O}_{2}$ & $\mathrm{Tp}$ & 54,3584 & 1 & 0,2 \\
\hline $\mathrm{Ar}$ & $\mathrm{Tp}$ & 83,8058 & 2 & 0,2 \\
\hline $\mathrm{Hg}$ & $\mathrm{Tp}$ & 234,3156 & 2 & 0,1 \\
\hline $\mathrm{H}_{2} \mathrm{O}$ & $\mathrm{Tp}$ & 273,16 & 0 (Definition Kelvin) & 0,04 \\
\hline $\mathrm{Ga}$ & $\mathrm{Sp}$ & 302,9146 & 1 & 0,1 \\
\hline $\mathrm{In}$ & $\mathrm{Ep}$ & 429,7485 & 3 & 0,5 \\
\hline $\mathrm{Sn}$ & $\mathrm{Ep}$ & 505,078 & 5 & 0,5 \\
\hline $\mathrm{Zn}$ & $\mathrm{Ep}$ & 692,677 & 13 & 0,7 \\
\hline $\mathrm{Al}$ & $\mathrm{Ep}$ & 933,473 & 25 & 1 \\
\hline $\mathrm{Ag}$ & $\mathrm{Ep}$ & 1234,93 & 40 & 2 \\
\hline $\mathrm{Au}$ & $\mathrm{Ep}$ & 1337,33 & 50 & 10 \\
\hline $\mathrm{Cu}$ & $\mathrm{Ep}$ & 1357,77 & 60 & 15 \\
\hline
\end{tabular}

Dd: Dampfdruck, Tp: Tripelpunkt, Sp: Schmelzpunkt, Ep: Erstarrungspunkt $u_{\text {thermodyn }}$ : Standardunsicherheit $(k=1)$ der thermodynamischen Temperatur $u_{\text {NMIs }}$ : typische Unsicherheit $(k=1)$ bei der Realisierung in Staatsinstituten (NMIs)

Als definierende Fixpunkte der ITS-90 sind thermodynamische Gleichgewichtszustände zwischen den Phasen reiner Substanzen festgelegt. Die zugrunde liegenden Zustände sind der Dampfdruck in Abhängigkeit von der Temperatur, Tripelpunkte, Erstarrungspunkte bzw. der Schmelzpunkt. Deren thermodynamische Temperaturen wurden für die ausgewählten Materialien an verschiedenen metrologischen Staatsinstituten mit höchster Genauigkeit bestimmt und unter Berücksichtigung der jeweils erzielten Messunsicherheiten gemittelt. Diese Mittelwerte waren die Grundlage für die Definition der Fixpunkte der ITS-90. Der entscheidende Vorteil dieser Vorgehensweise liegt darin, dass die Reproduzierbarkeit der ITS-90 wesentlich besser ist, als die Unsicherheit der Messung thermodynamischer Temperaturen. Hierduch kann sichergestellt werden, dass die Temperaturdarstellung weltweit einheitlich und die Stabilität der Skala auch über Zeiträume von Jahrzehnten gewährleistet ist. Bei Fixpunktzellen für den Temperaturbereich von $29,7646{ }^{\circ} \mathrm{C}(\mathrm{Ga})$ und $961,78(\mathrm{Ag})$ muss man zwischen geschlossenen Zellen und offenen Zellen unterscheiden. Geschlossenen Fixpunktzellen haben den Vorteil, dass diese gegen die Kontamination durch Verunreinigungen geschützt sind und mit vergleichsweise geringem technischen Aufwand betrieben werden können. Der wesentliche Nachteil geschlossener Zellen besteht darin, dass deren Innendruck nicht hinreichend genau bekannt ist. Hieraus resultuiert ein Unsicherheitsbeitrag welcher mehrere Millikelvin betragen kann. Daher werden geschlossenen Fixpunktzellen nicht für die Darstellung, sondern nur für die Weitergabe der Skala verwendet und müssen kalibriert werden. An Staatsinstituten verwedet man offene Zellen (Abb. 1). Diese können evakuiert, mit einem Schutzgas (Argon) gefüllt und dessen Druck während der Fixpunktrealisierung präzise gemessen werden. Eine weitere Anforderung an definierende Fixpunkte für die Darstellung der Skala ist die Kenntnis des Einflusses von Verunreinigungen auf die Schmelz- bzw. Erstarrungstemperatur. Dies erfordert bei Fixpunktmaterialien mit einer Reinheit von $99,9999 \%$ bis $99,99999 \%$ sowohl aus Sicht der chemischen Analytik, als auch in 
Bezug auf die Kenntnis der Phasendiagramme bei Verunreinigungskonzentrationen kleiner als 1 ppm, einen sehr hohen Aufwand [2]. Derartige Untersuchungen werden an Staatsinstituten durchgeführt, um die Einheitlichkeit der nationalen Skalenrealisierungen sicherzustellen.

\section{Die Interpolation zwischen den Fixpunkten}

Die Darstellung der Skala zwischen den Fixpunkten erfolgt durch Interpolation. In Abhängigkeit vom Temperaturbereich wurden hierfür unterschiedliche Messverfahren und Kennlinienverläufe festgeschrieben. So sind im Temperaturbereich oberhalb des Erstarrungspunktes von Silber $\left(961,78{ }^{\circ} \mathrm{C}\right)$ Strahlungsthermometer allen anderen Verfahren überlegen und werden daher als Interpolations- bzw. oberhalb des Kupferpunktes $\left(1084,62{ }^{\circ} \mathrm{C}\right)$ als Extrapolationsinstrument eingesetzt. Im Temperaturbereich von $13,8033 \mathrm{~K}$ bis $961,78{ }^{\circ} \mathrm{C}$ verwendet man hingegen PlatinNormalwiderstandsthermometer zur Interpolation der Skala. Da es jedoch kein PlatinNormalwiderstandsthermometer (SPRT) gibt, welches im gesamten Bereich eingesetzt werden kann, existieren verschiedene Typen bzw. Bauformen. So werden im Temperaturbereich von ca. $-200{ }^{\circ} \mathrm{C}$ bis $660{ }^{\circ} \mathrm{C}$ zumeinst Normalwiderstandsthermometer langer Bauart mit einem nominellen Widerstand von ca. $25 \mathrm{Ohm}$ bei $273,13 \mathrm{~K}$ eingesetzt. Bei tieferen Temperaturen verwendet man sogenannte KapselNormalthermometer. Diese sind bis ca. $+250{ }^{\circ} \mathrm{C}$ einsetzbar. Für Messungen oberhalb von $660{ }^{\circ} \mathrm{C}$ wurden Hochtemperatur-Normalthermometer entwickelt, für deren Messwiderstand Draht mit einem wesentlich größeren Durchmesser verwendet wird. Das Sensorelement hat einen nominellen Widerstand von etwa 0,25 Ohm bei 273,16 K. Um den Anwendern eine gewisse Flexibilität zu erlauben, wurden Unterbereiche definiert, in denen Interpolationsinstrumente unterschiedlicher Bauart oder sogar andere Verfahren verwendet werden dürfen. Hieraus resultiert zwar eine gewisses Maß an Mehrdeutigkeit bei der Darstellung und Weitergabe der Skala, aber die entsprechenden Unsicherheitsbeiträge sind vergleichsweise klein und gut bekannt.

\section{Die Weitergabe der ITS-90}

Die Weitergabe der ITS-90 erfolgt in Deutschland über die Physikalisch-Technische Bundesanstalt, akkreditierte Kalibrierlaboratorien und die Landeseichämter. Akkreditierungen von Kalibrierlaboratorien werden durch die Deutsche Akkreditierungsstelle $\mathrm{GmbH}$ (DAkkS) erteilt. "Akkreditierung ist die Bestätigung durch eine dritte Seite, die formal darlegt, dass eine Konformitätsbewertungsstelle die Kompetenz besitzt, bestimmte Konformitätsbewertungsaufgaben durchzuführen." (Definition gemäß DIN EN ISO/IEC 17011) [3]. Vereinfacht gesagt wird im Rahmen einer Akkreditierung überprüft ob ein Kalibrierlaboratorium in Bezug auf das Qualitätsmanagement und die technischen Anforderungen der ISO 17025 [4] genügt. Die Überprüfung der Fachkompetenz akkreditierter Laboratorien erfolgt in enger Zusammenarbeit mit der Physikalisch-Technischen Bundesanstalt (PTB), die gemäß Einheiten- und Zeitgesetz [5] für die Darstellung und Weitergabe der ITS-90 zuständig ist, und die Verantwortung für technische Fragestellungen hat. In Deutschland wird die ITS-90 in einem ersten Schritt von der PTB an akkreditierte Laboratorien weitergegeben. Dies erfolgt im technisch wichtigsten Temperaturbereich von ca. $-200{ }^{\circ} \mathrm{C}$ bis $1500{ }^{\circ} \mathrm{C}$ entweder mittels kalibrierter Fixpunktzellen, StandardPlatinwiderstandsthermometer, Edelmetallthermoelemente und Strahlungsthermometer, vereinzelt auch noch durch industrielle Widerstandsthermometer, FlüssigkeitsGlasthermometer und Thermistoren. 
Eine Kalibrierung von Thermometern an den Fixpunkten der ITS-90 ist nur für hochstabile und in ihrer Kennlinie genau bekannte Interpolationsinstrumente sinnvoll. Üblicherweise werden Kalibrierungen in akkreditierten Laboratorien mittels des Vergleichsverfahrens in gerührten Flüssigkeitsbädern durchgeführt. In derartigen Kalibrierbädern werden Werte für die Stabilität und Homogenität der Temperaturverteilung von wenigen Millikelvin erreicht, so dass durch den Vergleich mit einem kalibrierten, im gleichen Bad befindlichen Normalthermometer Unsicherheiten von 5 Millkelvin bei Raumtemperatur und (10-15) Millikelvin bei $600{ }^{\circ} \mathrm{C}$ erreicht werden. Der besondere Vorteil bei der Verwendung von Kalibrierbädern besteht darin, dass man hierin Thermometer unterschiedlichster Größe und Bauart bei optimaler thermischer Kontaktierung kalibrieren kann. Als Badflüssigkeiten verwendet man Alkohol, Wasser bzw. Mischungen von Wasser und Äthylenglykol, spezielle Silikon- oder Mineralöle und Salzmischungen. Hiermit werden Kalibrierungen von Berührungsthermometern im Temperaturbereich von $-80^{\circ} \mathrm{C}$ bis $600{ }^{\circ} \mathrm{C}$ durchgeführt. Bei höheren Temperaturen verwendet man entweder Sandbäder, spezielle Mehrzonenöfen mit Keramikausgleichsblock oder Wärmerohröfen. In der Tabelle 2 sind Thermometer und andere Kalibriergegenstände aufgeführt, für deren Kalibrierung Akkreditierungen durch die DAkkS erteilt worden sind. Dazu sind jeweils typische Temperaturbereiche und Messunsicherheiten angegeben. Im Unterschied zu den Angaben in Tabelle 1 sind hier die Messunsicherheiten entsprechend den Vorgaben für akkreditierte Laboratorien mit einem Erweiterungsfaktor $k=2$ angegeben. Dies bedeutet, dass der Wert der Messgröße im Regelfall mit einer Überdeckungswahrscheinlichkeit von $95 \%$ im zugeordneten Werteintervall liegt.

Tabelle 2 Kalibriergegenstände für die Messgröße Temperatur, für deren Kalibrierung DAkkS-Akkreditierungen erteilt wurden

\begin{tabular}{|c|c|c|}
\hline Kalibriergegenstand & typ. Temperaturbereich & typ. Messunsicherheit \\
\hline Fixpunktzellen & $-189{ }^{\circ} \mathrm{C}$ bis $962{ }^{\circ} \mathrm{C}$ & $1 \mathrm{mK}$ bis $10 \mathrm{mK}$ \\
\hline Normalthermometer & $-200{ }^{\circ} \mathrm{C}$ bis $962{ }^{\circ} \mathrm{C}$ & $3 \mathrm{mK}$ bis $15 \mathrm{mK}$ \\
\hline $\begin{array}{l}\text { industrielle } \\
\text { Widerstandsthermometer }\end{array}$ & $-200{ }^{\circ} \mathrm{C}$ bis $600^{\circ} \mathrm{C}$ & $5 \mathrm{mK}$ bis $30 \mathrm{mK}$ \\
\hline Thermoelemente & $0{ }^{\circ} \mathrm{C}$ bis $1560{ }^{\circ} \mathrm{C}$ & $0,3 \mathrm{~K}$ bis $2,5 \mathrm{~K}$ \\
\hline digitale Thermometer & $-20^{\circ} \mathrm{C}$ bis $300^{\circ} \mathrm{C}$ & $10 \mathrm{mK}$ bis $100 \mathrm{mK}$ \\
\hline Flüssigkeits-Glasthermometer & $-40{ }^{\circ} \mathrm{C}$ bis $500{ }^{\circ} \mathrm{C}$ & $10 \mathrm{mK}$ bis $1 \mathrm{~K}$ \\
\hline mechanische Thermometer & $-40^{\circ} \mathrm{C}$ bis $300^{\circ} \mathrm{C}$ & $0,5 \mathrm{~K}$ bis $5 \mathrm{~K}$ \\
\hline Oberflächenthermometer & $20^{\circ} \mathrm{C}$ bis $300{ }^{\circ} \mathrm{C}$ & $0,3 \mathrm{~K}$ bis $8 \mathrm{~K}$ \\
\hline $\begin{array}{l}\text { Temperatur-Indikatoren / - } \\
\text { Simulatoren }\end{array}$ & $-200{ }^{\circ} \mathrm{C}$ bis $1500^{\circ} \mathrm{C}$ & $1 \mathrm{mK}$ bis $20 \mathrm{mK}$ \\
\hline Temperatur-Blockkalibratoren & $-90^{\circ} \mathrm{C}$ bis $1200^{\circ} \mathrm{C}$ & $40 \mathrm{mK}$ bis $4 \mathrm{~K}$ \\
\hline Klimaschränke & $-40{ }^{\circ} \mathrm{C}$ bis $300{ }^{\circ} \mathrm{C}$ & $0,5 \mathrm{~K}$ bis $10 \mathrm{~K}$ \\
\hline Strahlungsthermometer & $-20{ }^{\circ} \mathrm{C}$ bis $3200{ }^{\circ} \mathrm{C}$ & $0,2 \mathrm{~K}$ bis $15 \mathrm{~K}$ \\
\hline
\end{tabular}

Beim Einsatz von kalibrierten Thermometern muss jedoch beachtet werden, dass zusätzliche, vom jeweiligen Messaufbau bzw. Verfahren abhängende Unsicherheitsbeiträge berücksichtigt werden müssen.

\section{Verfahren für die technische Temperaturmessung}

Bei der Auswahl eines geeigneten Temperaturmessverfahrens für praktische Anwendungen sollten die folgenden Kriterien berücksichtigt werden:

- In welchem Temperaturbereich soll gemessen werden? 
- Welche Messunsicherheit ist erforderlich?

- Welche anderen Randbedingungen sind einzuhalten: z.B. geometrische Vorgaben, dynamisches Verhalten, Robustheit (insbes. Vibrationsbeständigkeit), Drift- bzw. Alterungsverhalten, Einsatz in besonderen Umgebungen (radioaktive Bereiche, Explosionsschutz)? Neben Kenntnisen über die Vor- und Nachteile der verschiedenen Thermometertypen müssen auch anwendungsbedingte Unsicherheitsbeiträge berücksichtigt werden. Dies erfordert die Berücksichtigung von Wärmetransport- und Speicherprozessen, zugeschnitten auf den speziellen Anwendungsfall.

Die Beschreibung der Vielzahl von Verfahren für die Temperaturmessung würde den Rahmen dieses Beitrages sprengen, daher wird an dieser Stelle nur auf ausgewählte Methoden eingegangen und für weitere Informationen auf [6] verwiesen.

\section{Widerstandsthermometer}

Das zugrunde liegende Messprinzip ist die Temperaturabhängigkeit des elektrischen Widerstandes von Metallen, Halbleitern, Metalloxiden oder Kohlenstoff. Am häufigsten wird Platin verwendet, welches einen Temperaturkoeffizienten von ca. 0,4\%/K aufweist. Die weit verbreitenen Pt-100 Widerstandsthermometer sind genormt [7] und haben bei $0^{\circ} \mathrm{C}$ einen nominellen Widerstand von $100 \mathrm{Ohm}$. Thermometer die der IEC 60751 [7] entsprechen bezeichnet man als industrielle Widerstandsthermometer (IPRT). Es gibt drahtgewickelte Sensorelemente und sogenannte Dünnschichtsensoren. Im Vergleich zu Standard-Platinwiderstandsthermometern (SPRT) gibt es zwei wesentliche Änderungen. Dies sind die Verwendung von zusätzlichen Legierungsbestandteilen zur Einhaltung der genormten Kennlinie und konstruktive Maßnahmen zur Verbesserung der Vibrationsbeständigkeit. Die verbesserte Vibrationsbeständigkeit erreicht man durch eine stärkere Fixierung bzw. Einbettung des Platin-Messelementes. Der Nachteil besteht darin, dass Widerstandständerungen aufgrund mechanischer Spannungen (thermischer Längenänderungen) auftreten. Hieraus resultiert ein Hystereseverhalten, welches beim SPRT nicht vorhanden ist. Obwohl drahtgewickelte Pt-100 Sensoren den Dünnschichtelementen in Bezug auf die erreichbare Genauigkeit nach wie vor überlegen sind, haben sich Dünnschichtsensoren wegen ihres Preises und der geringen Fertigungstoleranzen durchgesetzt. Platin-Widerstandsthermometer sind im Temperaturbereich von ca. $-200{ }^{\circ} \mathrm{C}$ bis $1000{ }^{\circ} \mathrm{C}$ einsetzbar, ihr Hauptanwendungsbereich liegt aber unterhalb von ca. $500^{\circ} \mathrm{C}$.

Im Gegensatz dazu werden Thermistoren zumeist in einem sehr kleinen Temperaturbereich von ca. $-50{ }^{\circ} \mathrm{C}$ bis $+100{ }^{\circ} \mathrm{C}$ eingesetzt. Ihre Vorteile sind die wesentlich höhere Empfindlichkeit im Vergleich zu Pt-100 Sensoren und die kleinen geometrischen Abmessungen. Daher werden diese oft für medizinische Anwendungen verwendet.

\section{Thermoelemente}

Weil industrielle Widerstandtsthermometer bei höheren Temperaturen starken Alterungserscheinungen (Drift) unterliegen, dominieren im industriellen Bereich oberhalb von ca. $500{ }^{\circ} \mathrm{C}$ Thermoelemente. Das zugrunde liegende Messprinzip ist der Seebeck-Effekt, die Kennlinien der zehn gebräuchlichsten Thermoelementtypen sind ebenfalls normiert [8]. Aus Kostengründen werden bei industriellen Anwendungen zumeist Thermoelemente aus unedlen Metalle eingesetzt. Im Vergleich zu EdelmetallThermoelementen haben diese zwar eine höhere Empfindlichkeit sind aber wesentlich instabiler. So können z.B. im Temperaturbereich von $300{ }^{\circ} \mathrm{C}$ bis $600{ }^{\circ} \mathrm{C}$ für ein Typ $\mathrm{K}$ Thermoelement systematische Fehler von bis $\mathrm{zu} \pm 5 \mathrm{~K}$ auftreten, wohingegen mit einem Au/Pt-Thermoelement Unsicherheiten kleiner als $0,1 \mathrm{~K}$ erreichbar sind. Für 
Präzisionsanwendungen verwendet man daher grundsätzlich EdelmetallThermoelemente.

Bei hohen Temperaturen sind Strahlungsthermometer den Berührungsthermometern prinzipiell überlegen, die erreichbare Messunsicheit wird aber oft durch die unzureichende Kenntnis des Emissionsgrades limitiert.

\section{Rückführung, Kalibrierung und Eichung}

Die Anerkennung von Messergebnissen im Rahmen des Qualitätsmanagements (ISO 9001, ISO/IEC 17025, EN 45001) erfordert neben dem Einsatz von anerkannten Messverfahren auch die messtechnische Rückführung auf die Basiseinheiten des internationalen Einheitensystems. Für Temperaturmessungen beinhaltet dies die Rückführung auf die Einheit Kelvin und die Internationale Temperaturskala von 1990. Eine Rückführung bezieht sich auf eine Eigenschaft eines Messergebnisses oder den Wert eines Normals. Hierbei wird durch eine ununterbrochene Kette von Vergleichsmessungen (z.B. Kalibrierungen) mit gegebenen Messunsicherheiten ein direkter Bezug zu den Basiseinheiten des Internationalen Einheitensystems hergestellt. Bei der Kalibrierung eines Thermometers wird der damit gemessene Wert mit dem „wahren“ Wert verglichen, welcher mit einem Referenzgerät (z.B. Normalthermometer) bestimmt wird. Dazu gehört zwingend die Angabe einer Messunsicherheit. Als Ergebnis der Kalibrierung wird eine Abweichung zwischen dem gemessenen Wert und dem wahren Wert angegeben.

Im Unterschied dazu muss bei einer Eichung lediglich überprüft werden, ob der gemessene Wert innerhalb vorgegebener Eichfehlergrenzen [9] mit dem wahren Wert übereinstimmt. Hierbei ist es gängige Praxis, dass die verwendeten Verfahren und Geräte bei einer Kalibrierung und Eichung identisch sind. Ein weiterer Unterschied zwischen Eichung und Kalibrierung besteht darin, dass die Kalibrierung eine Momentaufnahme darstellt, bei einer Eichung hingegen Aussagen über das zeitliche Verhalten (Eichgültigkeitsdauer) gemacht werden.

\section{Zusammenfassung und Ausblick}

Temperaturmessungen werden auch in Zukunft im Vergleich mit Messungen anderer physikalischer Größen einen hohen Aufwand erfordern. Aufgrund der großen wirtschaftlichen Bedeutung und der Vielfalt der technischen Aufgabenstellungen ist auch weiterhin damit zu rechnen, dass immer neue Verfahren zur Temperaturmessung entwickelt werden. Diese haben für industrielle Anwendungen aber nur dann einen Wert, wenn der gemessenen Temperatur eine Messunsicherheit beigeordnet werden kann. Darüber hinaus ergibt sich aus Gründen des Qualitästmanagements die Notwendigkeit der messtechnischen Rückführung. In Deutschland ist diese durch die Physikalisch-Technische Bundesanstalt und ca. 70 durch die DAkkS im Bereich Temperatur akkreditierte Kalibrierlaboratorien und die Landeseichämter sichergestellt. Der mit einer Rückführung verbundene Aufwand hängt sehr stark vom Temperaturbereich, dem verwendeten Verfahren und der angestrebten Messunsicherheit ab. Darüber hinaus werden Kenntnisse über Wärmetransport- und Speicherprozesse benötigt, um anwendungsbezogenen Fehler zu minimieren.

\section{Literatur}

[1] H. Preston-Thomas, Metrologia 27 (1990) 3-10

[2] S. Rudtsch, T. Gusarova, A. Aulich, M. Fahr, J. Fischer, H. Kipphardt, 
R. Matschat, U. Panne, Int. J. Thermophys. 32 (2011), 293-302

[3] DIN EN ISO/IEC 17011, Konformitätsbewertung - Allgemeine Anforderungen an Akkreditierungsstellen, die Konformitätsbewertungsstellen akkreditieren, Beuth Verlag (2005)

[4] DIN EN ISO/IEC 17025, Allgemeine Anforderungen an die Kompetenz von Prüf- und Kalibrierlaboratorien, Beuth Verlag (2005)

[5] Gesetz über die Einheiten im Messwesen und die Zeitbestimmung http://www.gesetze-im-internet.de/me_einhg/index.html

[6] F. Bernhard, Technische Temperaturmessung, Springer Verlag Berlin, Heidelberg New York (2004)

[7] DIN EN 60751, Industrielle Platin-Widerstandsthermometer und Platin-Temperatursensoren, Beuth Verlag (2009)

[8] DIN EN 60584-1, Thermopaare - Teil 1: Grundwerte der Thermospannungen Beuth Verlag (1995)

[9] Gesetz über das Eich- und Messwesen http://www.gesetze-im-internet.de/eichg/index.html 